\title{
Assessment of bone metabolism and biomechanical properties of the femur, following treatment with anastrozole and letrozole in an experimental model of menopause
}

\author{
IOANNIS BOUTAS ${ }^{1,2}$, VASILIOS PERGIALIOTIS ${ }^{2}$, NICOLAOS SALAKOS ${ }^{1}$, LASKARINA-MARIA KOROU ${ }^{2}$, \\ ATHANASIOS MITOUSOUDIS ${ }^{3}$, EMMANOUIL KALAMPOKAS ${ }^{1}$, EFTHIMIOS DELIGEOROGLOU ${ }^{1}$, \\ ODYSSEAS GREGORIOU ${ }^{1}$, DESPINA N. PERREA ${ }^{2}$, GEORGE CREATSAS ${ }^{1}$ and STAVROS KOURKOULIS ${ }^{3}$
}

\author{
${ }^{1}$ Second Department of Obstetrics and Gynecology, University of Athens, 11528 Athens; \\ ${ }^{2}$ Laboratory of Experimental Surgery and Surgical Research 'N.S. Christeas', \\ National and Kapodistrian University of Athens Medical School, 11527 Athens; ${ }^{3}$ Department of Mechanics, \\ Laboratory of Testing and Materials-Unit of Biomechanics, National Technical University of Athens, 15773 Athens, Greece
}

Received September 7, 2016; Accepted January 17, 2017

DOI: $10.3892 / 01.2017 .6596$

\begin{abstract}
The aim of the present study was to investigate the impact of anastrozole and letrozole supplementation following surgically induced menopause on bone metabolism and biomechanical properties. A total of 45 Wistar rats underwent ovariectomy and were then randomly allocated to receive no treatment, anastrozole or letrozole. At 2 and 4 months following the initiation of the present study, the serum levels of osteoprotegerin (OPG) and receptor activator of nuclear factor- $\kappa \mathrm{B}$ ligand (RANKL) were determined, and the animals were sacrificed at the end of the 4-month period to assess the biomechanical properties of the femoral bones. The applied force and the deflection of the central section were recorded during the test. Taking advantage of these quantities, the fracture force, the stiffness of the bone and the energy absorbed until fracture were determined. At 2 months following the initiation of the experimental protocol, the mean OPG levels were significantly increased in the control group compared with the anastrozole-treated group $(\mathrm{P}<0.01)$. Similarly, RANKL levels were significantly increased in the control rats compared with the anastrozole-treated animals $(\mathrm{P}<0.001)$ and animals that received letrozole $(\mathrm{P}<0.05)$. Notably, these trends were not observed at
\end{abstract}

Correspondence to: Dr Ioannis Boutas, Second Department of Obstetrics and Gynecology, University of Athens, 76 Vas. Sofias Street, 11528 Athens, Greece

E-mail: ioannis.boutas@gmail.com

Abbreviations: AI, aromatase inhibitor; OPG, osteoprotegerin; RANKL, receptor activator of nuclear factor- $\mathrm{kB}$ ligand; control OVA, control ovariectomized group; OVA-A, ovariectomized anastrozole group; OVA-L, ovariectomized letrozole group; FDR, false discovery rate

Key words: anastrozole, letrozole, osteoporosis, RANKL, OPG, biomechanics the end of the experiment (4 months). A biomechanical study of the femoral bones revealed significantly decreased stiffness among animals that received anastrozole $(\mathrm{P}<0.05)$ and letrozole $(\mathrm{P}<0.01)$ compared with their control counterparts. The results of the present study indicate that treatment with anastrozole and letrozole significantly increases the levels of OPG and RANKL in bone, an effect that appears to be directly associated with the biomechanical properties of bones.

\section{Introduction}

Aromatase inhibitors (AIs) are commonly used drugs for the treatment of hormone-sensitive breast cancer in postmenopausal females (1). AIs limit the extra-ovarian production of estrogens, which occurs in fat, the liver and muscles via the aromatization of androgens. Third-generation AIs have increased selectivity and increased potency compared with earlier generation AIs (2).

Two types of AI have been developed based on their mechanism of action. Type I AIs are androgen analogues. Exemestane represents a unique third-generation steroidal AI that acts in an irreversible manner by binding to the substrate of the aromatase (3). Type II non-steroidal AIs, including anastrozole and letrozole, bind to the heme moiety of the enzyme in a reversible manner (4).

Among postmenopausal females who use these drugs, a major drawback is reductions in bone mass (5). AIs practically eliminate circulating estrogen levels, thereby increasing the risk of bone fracture in postmenopausal females (6). A randomized trial that was conducted in healthy postmenopausal females who received third-generation AIs demonstrated an increase in alkaline phosphatase, the serum collagen type I N-terminal propeptide and the resorption marker serum $\beta \mathrm{C}$-terminal crosslinking telopeptide of type I collagen, and a concomitant decrease in the parathyroid hormone compared with baseline measurements (7).

It is well known that AIs increase the risk of osteoporosis in postmenopausal females (8). However, little is known about 
the biomechanical characteristics of the osteoporotic bone and the presence of inflammation in these females $(9,10)$. Previous experimental studies in the field are scarce and conflicting. Goss et al (11) demonstrated that letrozole did not affect bone density and its mechanical properties in ovariectomized rats. Kumru et al (12) suggested that neither anastrozole nor letrozole had clear effects on bone mass density (both vertebral and femoral) of intact female rats. On the other hand, Yonden et al (13) observed that letrozole increased the risk of femoral fracture and of bone biomarkers, including alkaline phosphatase and phosphate.

In the present study, the biomechanical characteristics of the femoral bone were evaluated, and the levels of osteoprotegerin (OPG) and receptor activator of nuclear factor- $\kappa \mathrm{B}$ ligand (RANKL) were assessed in ovariectomized rats treated with anastrozole (OVA-A) and letrozole (OVA-L).

\section{Materials and methods}

Animals. A total of 45 female Wistar rats (Department of Animal Models for Biomedical Research, Hellenic Pasteur Institute, Athens, Greece) (4-week-old; mean body weight, $214.13 \mathrm{~g}$; range, 169-248 g) were placed in climate-controlled chambers (temperature, $20 \pm 1^{\circ} \mathrm{C}$; humidity, $55 \pm 5 \%$ ) under controlled lighting ( $12 \mathrm{~h}$ light/day) for 30 days to allow the animals to acclimate to their new environment. ELVIZ 510 food pellets (EL.VI.Z. S.A., Plati Imathias, Greece) containing full nutrient supplementation were provided ad libitum. Animal care and the surgical operations and the postoperative care were approved by the Athens University Medical School Ethics Committee and the Veterinary Directorate of Attica Region in agreement with Directive 2010/63/EU. On the night prior to the operation, the animals were not given access to food. At the final stage of the experiment, 10 control animals (female Wistar rats; mean body weight, $275.9 \mathrm{~g}$; range, 225-328 g) of a similar age (6 months) (control group without ovariectomy), which were treated in the same conditions as the experimental animals, were included.

Surgical procedures. A total of 45 female Wistar rats underwent surgical ovariectomy. The surgical procedures were performed between 8:00 a.m. and 9:00 a.m. on diestrus day 1 (T1). The animals were anesthetized with a combination of ketamine [75 mg/kg; Ketalar ${ }^{\mathrm{TM}}$ (ketamine hydrochloride), Pfizer, Inc., New York, NY, USA] and xylazine $\left(10 \mathrm{mg} / \mathrm{kg}\right.$; Rompun ${ }^{\mathrm{TM}}$; Bayer AG, Leverkusen, Germany) intraperitoneally. A midline dorsal skin incision was performed. The ovarian vessels were clamped and both ovaries were excised. The muscles and skin were sutured to close the incision.

Animal treatment. Following ovariectomy, the operated animals were randomized into 3 groups of 15 rats. The first group did not receive any drug regimen (control OVA), the second group received anastrozole (OVA-A) and the third group received letrozole (OVA-L) (Ariti SA, Acharnes, Greece). Administration of these regimens was performed according to the methodologies of a previous study (14). Specifically, anastrozole was administered orally in drinking water following dissolution in dimethyl sulfoxide solution at a concentration determined to result in a daily uptake of $\sim 0.1 \mathrm{mg} / \mathrm{kg}$ body weight; letrozole was similarly administered at a concentration determined to result in a daily uptake of $\sim 2 \mathrm{mg} / \mathrm{kg}$ body weight (12).

Blood samples were collected using capillary tubes from the medial retro-orbital venous plexus under light ether anesthesia (diethyl ether; Sigma-Aldrich; Merck Millipore, Darmstadt, Germany), at the beginning of the study (T1), at 60 days (T2) and at the end of the study (120 days; T3) at 9:00 a.m. after a 12-h fasting period.

At 4 months after the initiation of the present study, the animals were sacrificed by cervical dislocation while under ether anesthesia and sedation with intramuscular injection of $5 \mathrm{mg} / \mathrm{kg}$ xylazine (Rompun $^{\text {TM }}$; Bayer AG). The femoral bone was removed en bloc in order to perform the biomechanical study. At this point, 10 control animals (female Wistar rats; mean body weight, $275.9 \mathrm{~g}$; range, 225-328 g) of a similar age (6 months) (control group) were included in order to observe the potential biomechanical differences among the three groups compared with the biomechanical properties of wild-type bone. All animals were housed under similar conditions.

Enzyme-linked immunosorbent assay (ELISA). Following sampling, the blood specimens were transferred to Vacutainer tubes (BD Biosciences, Franklin Lakes, NJ, USA). The serum was separated following centrifugation of the blood at $1500 \mathrm{xg}$ at $4^{\circ} \mathrm{C}$ for $10 \mathrm{~min}$. The specimens were stored at $-30^{\circ} \mathrm{C}$ until the assay, which was performed within 2 months. Serum OPG and RANKL levels were measured using commercially available kits (OPG; catalog no. SEA108Ra; Uscn Life Sciences, Inc., Wuhan, China; RANKL; catalog no. SEA855Ra; Uscn Life Sciences, Inc.) according to the manufacturer's protocol. All measurements were performed using an ELISA photometer (Model 680 Microplate Reader; Bio-Rad Laboratories, Inc., Hercules, CA, USA). The samples were tested in duplicate, and the plates were read at $450 \mathrm{~nm}$.

Biomechanical study. The experimental set-up for biomechanical study included an electromechanical loading frame (10 kN MTS INSIGHT ${ }^{\text {тм }}$ Material Testing System; MTS Systems Corporation, Eden Prairie, MN, USA), which was specifically designed for biomechanical tests, a Real Time Strain Sensor video extensometer (Limess Messtechnik and Software GmbH, Krefeld, Germany), a digital camera, suitable grips for three-point bending tests with small specimens, digital calipers and a personal computer equipped with the appropriate software (TestWorks ${ }^{\circledR} 4$, MTS Systems Corporation, Eden Prairie, MN, USA).

A series of preliminary tests (Fig. 1A) were conducted to calibrate the experimental set-up concerning the ranges of force and deflection and also the loading rate. During the preliminary tests, a plastic cylinder (diameter, $2 \mathrm{~mm}$ ) was attached at the upper wedge of the frame (Fig. 1A) to avoid local damage to the bone (the punch effect). This approach was abandoned in the main experimental protocol because the reduced stiffness of the plastic inevitably influenced the data associated with the deflection. In the main experimental protocol, the specimens were in direct contact with both the supporting and loading metallic elements of the loading fixture (Fig. 1B). The femurs were placed horizontally on rounded edges with a radius of curvature of $3 \mathrm{~mm}$ at a distance 



Figure 1. (A) Typical specimen of the preliminary tests. (B) Typical specimen of the main experimental protocol during fracturing. (C) Typical specimen as seen through the screen of the video extensometer just prior to the onset of loading.

of $20 \mathrm{~mm}$. Care was taken to place all specimens exactly in the same orientation to minimize scattering factors. The force was applied at the mid-section of the diaphysis using a punch with a rounded notch (Fig. 1B). Fig. 1C presents a typical specimen observed on the screen of the video extensometer immediately prior to the onset of the loading procedure.

The displacement control loading mode was selected. The rate of the imposed displacement was selected to be equal to $0.5 \mathrm{~mm} / \mathrm{min}$ in an attempt to simulate quasi-static loading conditions. The displacement was imposed continuously until fracture.

The force was applied monotonically and the test was considered to be completed when the specimen fractured. The raw data recorded during the biomechanical test included the applied force (measured with the aid of a calibrated load cell with a sensitivity of $0.1 \mathrm{~N}$ ) and the deflection of the mid-section of the specimens measured using the video extensometer (with a distance resolution of $0.5 \mu \mathrm{m}$ for a $100-\mathrm{mm}$ field of view).

The fracture of all specimens was brittle, without exception. Typical force-deflection curves, obtained during the tests, are presented in Fig. 2. These curves are generally characterized by an initial non-linear portion, which is followed by an almost perfectly linear portion and a non-linear portion (the exact shape of the curve depends on the failure mode of the specific specimen). Apart from the similarities between the force-deflection curves, a number of differences in the shapes of the curves were noted. These differences dictated the classification of the tests into three categories, as presented in Fig. 2. The majority of the tests were classified in the first category (Fig. 2A) (i.e., the force-displacement curve was monotonous during the entire duration of the tests). A limited number of specimens were classified in the second category (Fig. 2B), which included tests for which the force continued to increase for a time prior to the specimen collapsing following initial cracking of the specimen (denoted by a more or less sudden load-drop). Two of the specimens were classified in the third category, which included tests for which the main force-deflection curve consisted of two linear segments (Fig. 2B). These segments were characterized by the same slope. Analysis of the images captured during the specific tests revealed that the discontinuity of the curve was due to a sudden rotation of the specimen about its longitudinal axis. Considering that the slope of the two segments of the force-deflection curve was the same, the specimens in the specific category were not excluded from the analysis and were considered to be successful.

Based on the raw data, the following quantities were determined following the tests: i) The fracture force $(\mathrm{N})$ sustained by the bone specimen. For the specimens in the first and the third categories, the fracture force was defined as the actual maximum force recorded (denoted as the peak force in Fig. 2A and $\mathrm{C}$ ). For the specimens in the second category, the fracture force was considered to be the force at the initial crack (again, denoted as the peak force in Fig. 2B). ii) The stiffness of the bone $(\mathrm{N} / \mathrm{mm})$ was quantified as the slope of the linear portion of the force-deflection curve. For all three categories, ignoring an initial non-linear region (which was systematically observed in almost all of the specimens and which was attributed to micro-displacements of the specimen until perfect contact with the supports was achieved), the linear portion was clearly distinguishable and ranged between 5 and $70 \%$ of the peak force. iii) The energy absorbed by the specimen until fracture $(\mathrm{N} \cdot \mathrm{mm})$. The energy was defined as the area below the force-deflection curve (the dashed area in Fig. 2). For the first two categories, this quantity was calculated via numerical integration of the force-deflection curve (obtained through a proper curve-fitting procedure using commercially available software (MATLAB version R2010b; Mentor Hellas Ltd., Pallini, Attiki, Greece) from 0 up to the fracture force (i.e., the peak force). For the third category of tests, the procedure was similar. However, the area corresponding to the rigid-body d isplacement due to the specimen's rotation (the shaded area in Fig. 2C) was removed as it was thought to not correspond to actual energy absorption.

From a biomechanics point of view, the fracture force (although an easily determined quantity) is not the most appropriate quantity for the characterization of strength, particularly for comparative purposes, as it may depend on several factors, including the geometrical characteristics of the fractured cross-section (15). In order to cope with this problem, the second moment of area of the specimens was calculated by taking advantage of data pertaining to the geometric features of the cross-sections. 

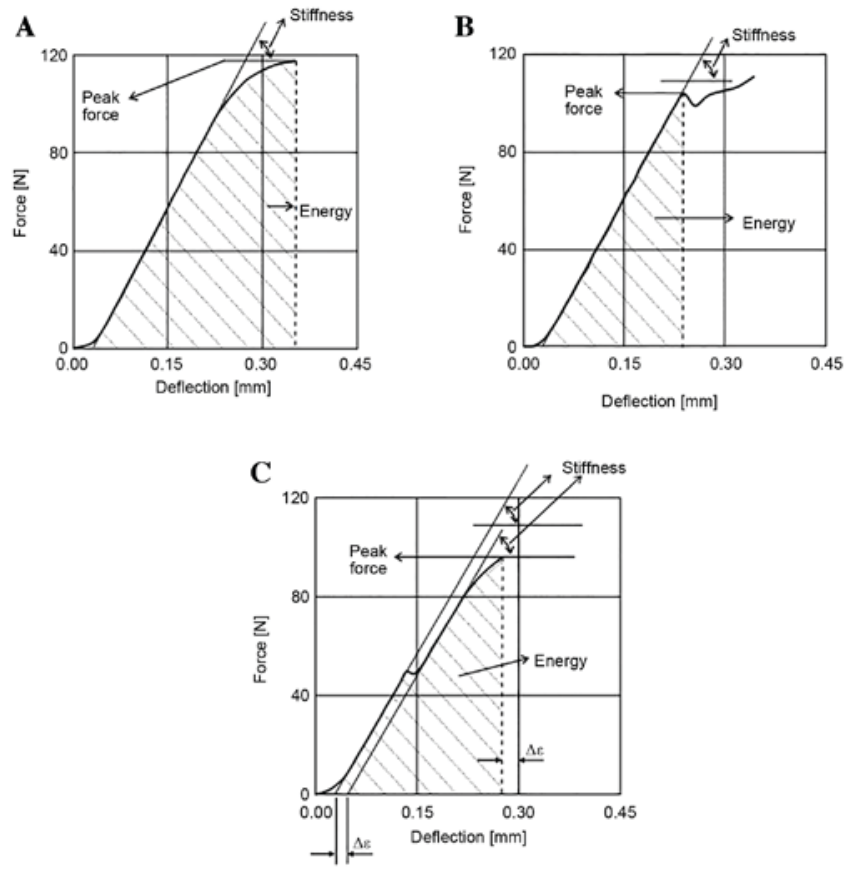

Figure 2. Typical force-displacement curves of the three test categories: (A) First category; (B) second category; (C) third category.

Therefore, one of the two pieces of the fractured bones (stored at $-18^{\circ} \mathrm{C}$ ) was placed vertically in a small cup using a specially designed metallic structure (Fig. 3A) that was filled with molten resin (Fig. 3B). Following a period of curing, the resin solidified and the resin-bone complex was removed from the cup. Its upper surface was smoothed via abrasion to remove the minimum possible amount of material from the fractured bone surface until a planar surface was obtained. Using a stereoscope and a digitizer, the outer and inner boundaries of the fractured surface of the bone were obtained (Fig. 3C and D). The geometrical features of the cross-sections of the fractured bone sections were obtained. These features are shown in Fig. 3E and included: i) The co-ordinates of the centroid, $\mathrm{C}$, of the cross-section with respect to an arbitrary reference system, ii) the centroidal principal axes of the second moment of area $\mathrm{Cy}_{\mathrm{p}}$ and $\mathrm{Cz}_{\mathrm{p}}$ and iii) the principal second moments of area, $\mathrm{I}_{\mathrm{ypyp}}$ and $\mathrm{I}_{\mathrm{zpzp}}$.

It was determined that the average values of the principal second moments of area of the various groups of specimens were almost identical. The differences in any case were within the accepted level of experimental scattering; the differences calculated were $<2.65 \%$ for any combination of specimen groups.

The next decisive step in this direction was to calculate the fracture stress (bending strength) using the following formula:

$$
\sigma_{x x, \text { fracture }}= \pm \frac{P_{\text {peak }} \cos \left|\omega_{p}\right| \frac{L}{4}}{I_{z_{\mathrm{p}} z_{\mathrm{p}}}} y_{c} \frac{P_{\text {peak }} \sin \left|\omega_{p}\right| \frac{L}{4}}{I_{y_{p} y_{p}}} z_{c}
$$

where $I_{z_{p} z_{p}}$ and $I_{y_{p} y_{p}}$ are the principal second moments of area, $P_{\text {peak }}$ is the fracture force (defined as previously described and assuming that the linearity assumption is valid), $\omega_{\mathrm{p}}$ is the angle between the principal $y_{\mathrm{p}}$-axis and the respective loading axis
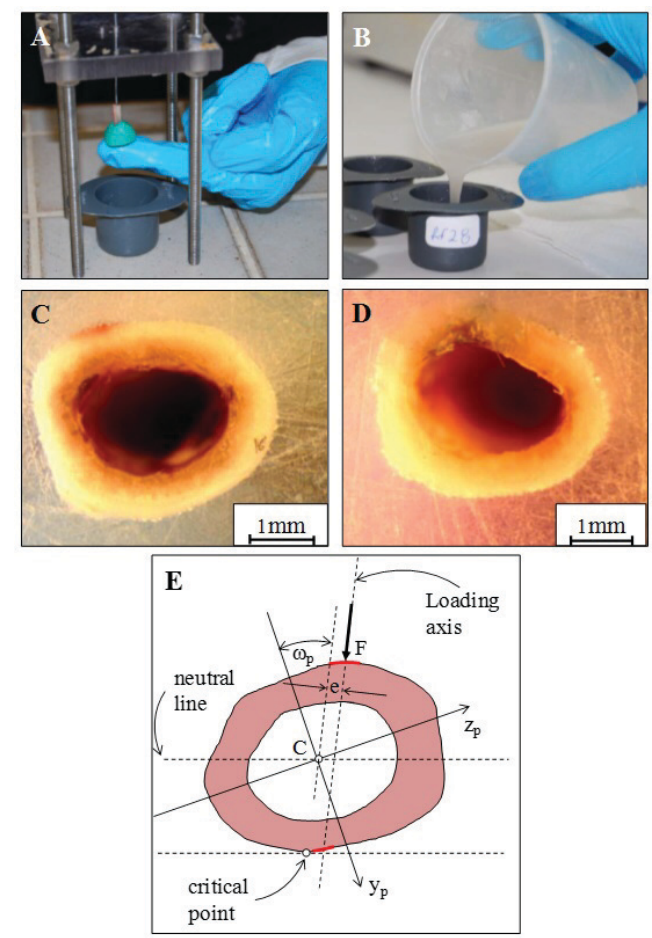

Figure 3. Procedure used to obtain the characteristics of the fractured cross-sections. (A) The fractured bone was placed vertically in a cup. (B) Molten resin was poured into the cup. (C and D) Cross-sections of typical fractured specimens following abrasion. (E) Geometric features of the fractured cross sections required for the determination of fracture stress: $\mathrm{C}$ is the centroid of the cross section, $\mathrm{Cy}_{\mathrm{p}}$ and $\mathrm{Cz}_{\mathrm{p}}$ are the centroidal principal axes of the second moment of area, $\mathrm{e}$ is the eccentricity (distance between the axis of the force $\mathrm{F}$ and a parallel axis passing from the centroid) and $\omega_{\mathrm{p}}$ is the angle between the principal $\mathrm{y}_{\mathrm{p}}$-axis and the loading axis.

(Fig. 2), and $y_{\mathrm{c}}$ and $z_{\mathrm{c}}$ are the coordinates of the critical point (the most stressed one) of the fractured section with respect to the principal system. However, determining $\sigma_{z z \text {,fracture }}$ is not a trivial task (even in cases in which the geometry of the cross-section is appropriately described). $\sigma_{z z \text {,fracture }}$ depends on the orientation of the loading axis with respect to the principal centroidal axes (i.e., the angle $\omega_{\mathrm{p}}$ ), which, in turn, depends on small-scale anatomical details of the bone that dictate the exact position of the bone on the supports, the exact position of the critical point $\left(y_{\mathrm{c}}, z_{\mathrm{c}}\right)$ and the possible distance of the loading axis from the centroid of the cross-section. Preliminary research in progress indicates that at least the last term is small and may be ignored; however, it is not yet possible to make definite conclusions.

As the differences in the principal second moments of area of the fractured cross-sections of the specimens between the various groups are not statistically significant, it is reasonable, at least as a first approximation, to assume that the fracture force is a criterion of the load-carrying capacity of the bone as a structural element.

Statistical analysis. Results were expressed as the mean \pm standard deviation for continuous normally distributed variables and as the median and interquartile range for non-symmetric data. The normality of the distributions was assessed using the Kolmogorov-Smirnov test and graphical methods. 

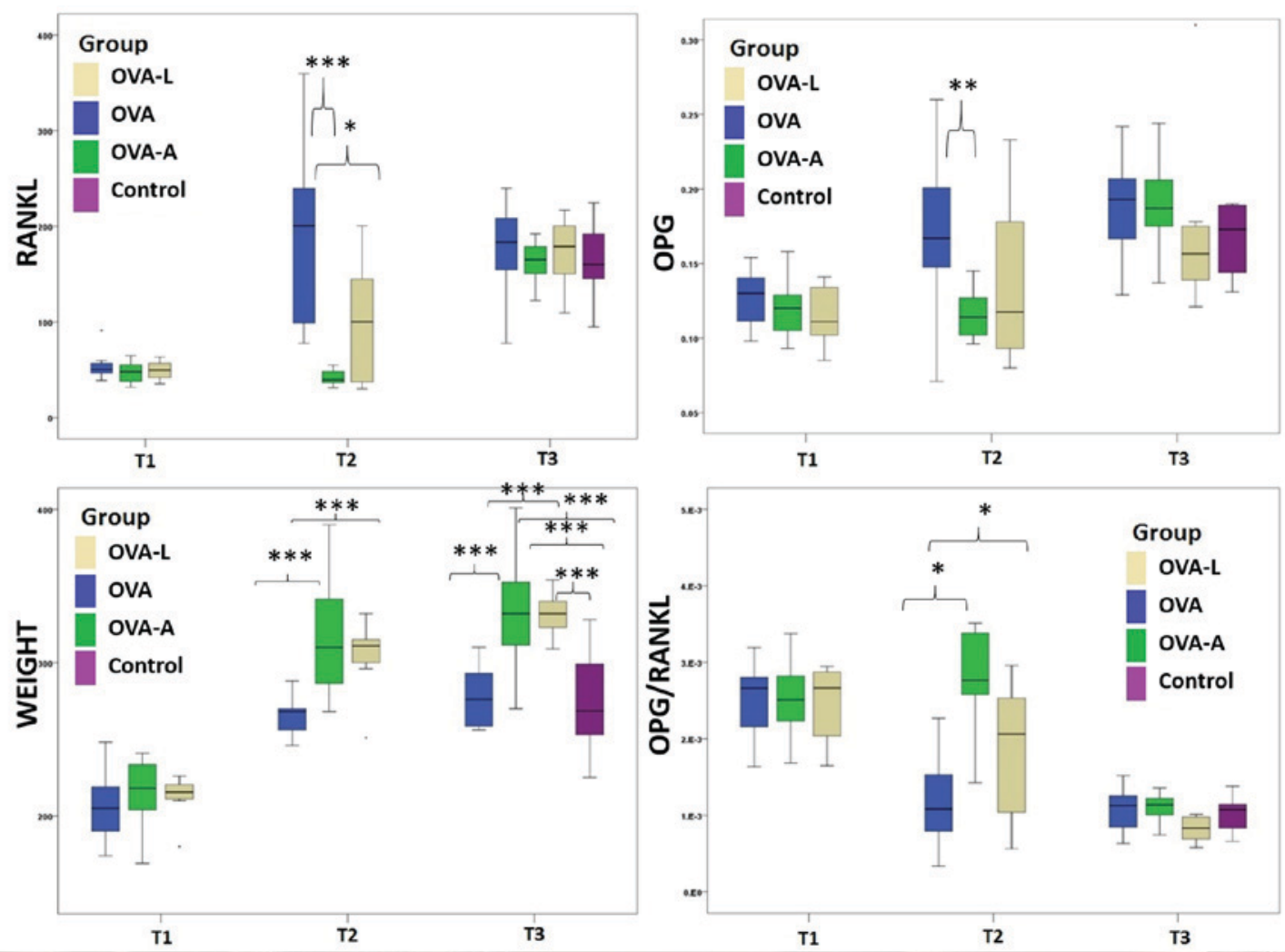

Figure 4. Box plots displaying the second and third quartiles, as well as the median of serum RANKL (pg/ml) levels, serum OPG (ng/ml), body weight levels (g) and OPG/RANKL ratio at baseline in the groups studied. The groups were: Control group (without ovariectomy; $\mathrm{n}=10$ ), control OVA group (with ovariectomy; $\mathrm{n}=15)$, OVA-A group ( $\mathrm{n}=15)$ and OVA-L group ( $\mathrm{n}=15)$, at baseline (T1), 2 months (T2) and 4 months (T3). RANKL, receptor activator of nuclear factor- $\kappa \mathrm{B}$ ligand; OPG, osteoprotegerin; OVA, ovariectomized; A, anastrozole group; $\mathrm{L}$, letrozole. ${ }^{*} \mathrm{P}<0.05,{ }^{* *} \mathrm{P}<0.01,{ }^{* * *} \mathrm{P}<0.001$.

Comparisons between $>2$ groups were performed using analysis of variance (ANOVA). The Kruskal-Wallis test was used as a non-parametric test for multiple group comparisons, and the Mann-Whitney U test was used for post hoc multiple testing. Comparisons between multiple time points were performed using repeated-measures ANOVA; Friedman's test with Wilcoxon's signed ranks test was used for post hoc comparisons. Pearson's correlation coefficient and Spearman's rho (r) were calculated to assess associations between variables. In all cases of multiple hypothesis testing, Benjamini-Hochberg's false discovery rate (FDR) was used to assess differences and to control family-wise errors to be $<0.05$. All tests were two-sided. $\mathrm{P}<0.05$ was considered to indicate a statistically significant difference. Statistical analysis was performed using the SPSS statistical package (IBM SPSS, version 22.0; Armonk, NY, USA).

\section{Results}

The baseline serum levels of OPG and RANKL were comparable among the three groups of animals analyzed in the present study (Fig. 4). At 2 months following the initiation of the present study, the mean OPG levels were increased in the control OVA group compared with the OVA-A group $(\mathrm{P}<0.01$; Fig. 4). Similarly, the levels of RANKL were also increased in the animals belonging to the control OVA group compared with the OVA-A group ( $\mathrm{P}<0.001$; Fig. 4) and the OVA-L group $(\mathrm{P}<0.05 ;$ Fig. 4). However, no significant difference in RANKL and OPG levels at the end of the experiment (4 months) was identified (Fig. 4). OPG/RANKL ratio was increased at T2 in OVA-A and OVA-L groups when compared with the OVA group ( $\mathrm{P}<0.05$ in all cases).

The baseline mean body weights among the study groups were comparable (Fig. 4). At 2 months following ovariectomy, the body weights in the control OVA group were significantly decreased compared with the body weights of the OVA-A and OVA-L groups ( $\mathrm{P}<0.001$ in all cases; Fig. 4). At the end of the experiment (4 months), the OVA-A and OVA-L groups had increased body weights relative to the control or control OVA animals ( $\mathrm{P}<0.001$ in all cases; Fig. 4). Table I presents all the biochemical parameters.

The biomechanical study of the femoral bones revealed a decreased stiffness in the OVA-A and OVA-L groups compared with the control animals $(\mathrm{P}<0.01)$ and with the control OVA groups $(\mathrm{P}<0.05$; Fig. 5). It was observed that anastrozole and letrozole significantly affected the stiffness of bone $(\mathrm{P}=0.0135$; ANOVA; following Benjamini-Hochberg's FDR correction for multiple hypothesis testing).

Principal second moment analysis revealed that the differences in the mean values of the principal second moment of area of the various groups of specimens were negligible and in any case within the accepted experimental scattering; the differences were $<2.65 \%$ for any combination of specimen groups.

Association statistics revealed a positive association between fracture force and energy absorbed $(r=0.7 ; \mathrm{P}<0.001)$, as well as between fracture force and bone stiffness $(r=0.38$; $\mathrm{P}<0.05$ ). Bone stiffness was also positively associated with the 
Table I. Body weight levels, serum RANKL, serum OPG levels and serum OPG/RANKL ratio at T1, T2 and T3.

\begin{tabular}{|c|c|c|c|c|c|c|}
\hline \multirow[b]{2}{*}{ Measurement } & \multirow[b]{2}{*}{ Time } & \multicolumn{4}{|c|}{ Group } & \multirow{2}{*}{$\begin{array}{c}\text { Differences } \\
\text { between groups }\end{array}$} \\
\hline & & Control & OVA & OVA-A & OVA-L & \\
\hline \multirow[t]{3}{*}{ Body weight, g } & $\mathrm{T} 1$ & N/A & $215(34.00)$ & $218.00(30.00)$ & $215.5(10.25)$ & NS \\
\hline & $\mathrm{T} 2$ & N/A & $259.00(23.00)$ & $304.00(38.00)$ & $311.00(15.00)$ & $\begin{array}{l}\text { OVA vs. OVA-A } \\
\text { OVA vs. OVA-L }\end{array}$ \\
\hline & $\mathrm{T} 3$ & $268.50(53.50)$ & $276.00(41.00)$ & $332.00(56.00)$ & $332.00(18.50)$ & $\begin{array}{l}\text { OVA vs. OVA-A }{ }^{a} \\
\text { OVA vs. OVA-L }{ }^{a}\end{array}$ \\
\hline \multirow[t]{3}{*}{ Serum RANKL levels, $\mathrm{pg} / \mathrm{ml}$} & $\mathrm{T} 1$ & N/A & $50.32(13.58)$ & $47.88(20.73)$ & $49.71(17.53)$ & NS \\
\hline & $\mathrm{T} 2$ & N/A & $200.45(144.88)$ & $39.44(18.76)$ & $100.09(133.77)$ & $\begin{array}{l}\text { OVA vs. OVA-A } \\
\text { OVA vs. OVA-L }\end{array}$ \\
\hline & $\mathrm{T} 3$ & $160.28(55.04)$ & $183.42(74.47)$ & $165.15(37.75)$ & $178.94(52.78)$ & NS \\
\hline \multirow[t]{3}{*}{ Serum OPG levels, ng/ml } & $\mathrm{T} 1$ & N/A & $0.13(0.03)$ & $0.12(0.03)$ & $0.11(0.033)$ & NS \\
\hline & $\mathrm{T} 2$ & N/A & $0.16(0.09)$ & $0.11(0.05)$ & $0.11(0.09)$ & OVA vs. OVA-A ${ }^{\mathrm{a}}$ \\
\hline & $\mathrm{T} 3$ & $0.17(0.05)$ & $0.19(0.06)$ & $0.19(0.04)$ & $0.15(0.04)$ & NS \\
\hline \multirow[t]{3}{*}{ Serum OPG/RANKL ratio } & $\mathrm{T} 1$ & N/A & $0.002(0.00)$ & $0.002(0.00)$ & $0.002(0.00)$ & NS \\
\hline & $\mathrm{T} 2$ & N/A & $0.001(0.00)$ & $0.002(0.00)$ & $0.002(0.00)$ & $\begin{array}{l}\text { OVA vs. OVA-A } \\
\text { OVA vs. OVA-L }\end{array}$ \\
\hline & $\mathrm{T} 3$ & $0.001(0.00)$ & $0.001(0.00)$ & $0.001(0.00)$ & $<0.001(0.00)$ & NS \\
\hline
\end{tabular}

The data are presented as the median (interquartile range). The groups were: Control group ( $\mathrm{n}=10)$, control OVA group ( $\mathrm{n}=15$ ), OVA-A group $(\mathrm{n}=15)$ and OVA-L group $(\mathrm{n}=15) .{ }^{\mathrm{a}} \mathrm{P}<0.05$. OVA, ovariectomized; A, anastrozole group; L, letrozole group; T1, baseline; T2, 2 months after surgery; T3, 4 months after surgery; N/A, not applicable; NS, not significant.

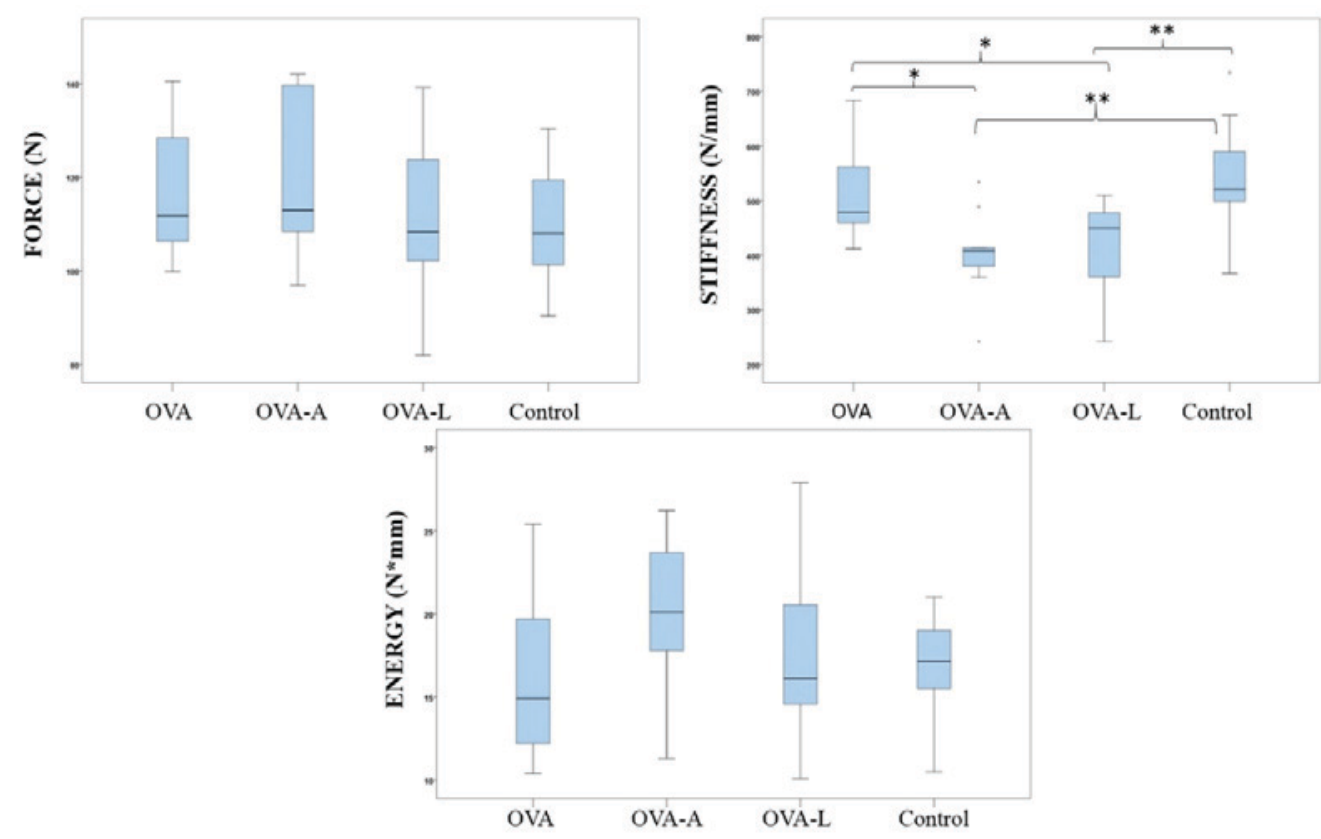

Figure 5. Box plots displaying the second and third quartiles, as well as the median of the fracture force (N), energy absorbed until fracture (N·mm), stiffness of the bones $(\mathrm{N} / \mathrm{mm})$ as obtained from the biomechanical study of the femur of the animals of the experimental groups at 4 months. The groups were: Control group (without ovariectomy; $n=10$ ), control OVA group (with ovariectomy; n=15), OVA-A group (n=15) and OVA-L group (n=15). OVA, ovariectomized; A, anastrozole group; L, letrozole. ${ }^{*} \mathrm{P}<0.05,{ }^{* *} \mathrm{P}<0.01$.

basal serum RANKL levels ( $\mathrm{r}=0.36$; $\mathrm{P}<0.05)$, as well as with RANKL levels 2 months later $(\mathrm{r}=0.49 ; \mathrm{P}<0.05)$. However, a negative association between bone stiffness and the rats' body weights was observed two months after $(\mathrm{r}=-0.39 ; \mathrm{P}<0.05)$.
A negative association was also noted between body weight increase during the transition from the second month until the completion of the study $(\mathrm{r}=-0.48 ; \mathrm{P}<0.05)$. A positive association between serum RANKL and OPG levels $(r=0.7 ; \mathrm{P}<0.001)$ and a 
significant negative association of the OPG/RANKL ratio with body weight $(r=-0.36 ; \mathrm{P}<0.001)$ were additionally recorded.

\section{Discussion}

Estrogens are derived from the aromatization of circulating androgens. Aromatase, a member of the cytochrome P450 superfamily enzymatic complex, is responsible for the conversion of C-19 androgen into C-18 estrogen (15). It is expressed in numerous tissues, including adipose tissue, liver, muscle, breast, brain and fibroblasts (16-18). AIs are frequently used in postmenopausal breast cancer patients as an adjuvant treatment when the tumor is estrogen-dependent (19). However, their use has been reported to induce accelerated bone loss via the depletion of circulating estrogen $(6,20)$. In the present study, the potential differences among control non-ovariectomized and ovariectomized Wistar rats that received letrozole (OVA-L), anastrozole (OVA-A) or in the control OVA group were investigated. In this context, levels of two well-defined bone markers (the osteoclastogenic cytokine RANKL and its decoy receptor OPG) that have been extensively investigated within the last decade $(21,22)$ were determined. In addition, the biomechanical characteristics of femoral bones following treatment with AIs were also determined, a parameter that, to the best of our knowledge, has not previously been studied.

Results of the present study and relevance to other studies in the field. The results of the present study indicated that administration of letrozole and anastrozole to rats following the surgical induction of menopause did not appear to be to associated with altered serum OPG or RANKL levels. However, a significantly reduced OPG/RANKL ratio was observed among ovariectomized animals that did not receive AI. A number of biomarkers are associated with bone turnover, including RANKL and OPG, which have been investigated previously $(23,24)$. Activated osteoclasts participate in bone loss and the development of erosions, and RANKL stimulates osteoclasts. On the other hand, inhibition of osteoclasts and the activation of osteoblasts are associated with bone formation and ossification (25) OPG, which is produced by osteoblasts, inhibits RANKL and leads to bone formation (26). The OPG/RANKL ratio is a key regulator of bone metabolism that can adequately determine bone mass and bone integrity $(27,28)$. In particular, it seems to be more relevant compared with OPG alone as a marker of bone resorption that exerts biological effects on osteoclasts and osteoblasts $(19,29)$. The activation of RANKL is associated with bone loss. On the other hand, OPG inhibits RANKL and supports bone formation. Therefore, a decreased OPG/RANKL ratio favors bone loss. The OPG/RANKL ratio has been associated previously with body weight, a parameter that typically influences physical development and bone integrity $(30,31)$. OPG and RANKL measurements exhibited variation for the control OVA and OVA-L groups at T2. However, the variance and values for the OVA-A group at T2 were comparable with those at $\mathrm{T} 1$, which indicates that there was no systemic response in rats. The control OVA group exhibited significant variance in RANKL and OPG levels. However, in spite of variation in the magnitude of increase in this group, all mice at least doubled their RANKL measurements. In the OVA-L group at T2, half of the animal models exhibited increased RANKL levels, and the other half maintained the baseline levels. However, at $\mathrm{T} 3$ the within-group variances were decreased and almost all of the animals increased their RANKL measurements to a similar level. This increase began at different time points for each group: Prior to T2 for the control OVA group, at around T2 for the OVA-L group and later on for the OVA-A group. It is likely that the late onset of increased RANKL levels in the OVA-A group is beneficial to femur structure and biomechanical properties.

From a biomechanics perspective, anastrozole and letrozole do not appear to significantly affect a bone's fracture force and the respective energy absorbed. However, it was observed that anastrozole and letrozole significantly affected the stiffness of bone ( $\mathrm{P}=0.0135$, ANOVA, following Benjamini-Hochberg's FDR correction for multiple hypothesis testing). Based on previous studies, stiffness determines bone deformation and is directly associated with a bone's strength (32-34). In a previous study, Zioupos and Currey (35) demonstrated that aging has a detrimental effect on the elastic and ultimate properties of cortical bone. These authors also stated that a $2.3 \%$ decrease in stiffness was directly associated with a $4.1 \%$ reduction in fracture toughness (35). In the present study, the fracture force was identified to be directly associated with stiffness, an observation that may partially explain the increased tendency of bones exposed to AI treatment to develop fractures.

Vegger et al (36) studied the mechanical properties of the femur bone using a three-point bending test in rats treated with strontium ranelate. In their study, a femur was placed in a specific implement by supporting the ends of the bone, and the maximum load and stiffness were determined. Naruse et al (37) performed a three-point bending test in ovariectomized osteoporotic rats to study the effect of alendronate in femur bones and evaluate the mechanical properties. Gasser et al (38) performed an ovariectomy in female Wistar rats and studied the effects of AIs on the biomechanical properties of distal femoral metaphysis using a three-point bending test. These authors identified that femoral metaphysis bone strength and stiffness were significantly decreased in the control OVA and the OVA-L groups, with a greater decrease in the control OVA group. In addition, a significant increase in RANKL was observed in the control OVA and the OVA-L group.

The results of the present study have demonstrated that the administration of anastrozole or letrozole to ovariectomized rats affects the stiffness of femurs. At 2 months following ovariectomy, serum OPG levels were higher in the control OVA group than in the OVA-A group. OPG enhances osteogenesis. As a consequence, these results are consistent with the outcomes of a biomechanical study conducted using femoral bones. Paradoxically, the control OVA group, at the same time, exhibited significantly higher serum RANKL levels that stimulated osteoclast differentiation and therefore bone resorption compared with the OVA-A and OVA-L groups.

Strengths and limitations of the present study. The primary strength of the present study was its randomized nature and the assessment of the biomechanical characteristics of the femurs; these aspects were combined with an investigation of serum RANKL and OPG levels. Furthermore, the 4-month course of AI treatment in rats corresponds to $\sim 6.5$ years in humans, 
which is an adequate period of time to investigate the expected effects of AIs on bone turnover (39). The present study is partly limited; however, by a lack of additional pathological analysis because of the lack of access to a sledge microtome.

A limitation to the study design was the lack of a sham-operated control group, which would have enabled the incorporation of surgical stress and inflammation into the measured parameters. The current design comprised two distinct controls [i.e., untreated ovariectomized animals and non-ovariectomized untreated controls (age- and sex-matched rats)]. These two groups enabled the assessment of the effect of the different treatments on biomarkers and biomechanical properties, as well as providing reference values for the measured parameters. However, the two groups did not permit the evaluation of the effects of the surgical procedure, as well as the administration vehicle, on the relevant measurements. The present study focused primarily on alterations in biomarkers and mechanical properties occurring at the end point of its 4-month protocol, and it also yielded measurements at two further time points.

Implications for future research. Given the possibility that significant alterations may have occurred in biomarker levels and bone mechanical properties from the transition between 0 and 4 months, it seems plausible to assume that the inclusion of additional time points is necessary in future studies. Furthermore, the inclusion of other biomarkers of bone metabolism (including parathormone, $\beta_{2}$-adrenergic receptors, insulin growth factor-1 and fibroblast growth factors) may reveal other facets of the underlying molecular mechanisms.

Although the present study was intended to assess the impact of anastrozole and letrozole on the biomechanical properties of the femoral bone by determining the fracture force, the stiffness of the bones and the energy absorbed until fracture, ultrasound computed tomographic analysis of bones as well as their histological or histomorphometric analysis may provide valuable insight into the effects of AIs on bone structure and metabolism. This parameter should be taken into account in future relevant studies.

Furthermore, the association of biomechanical abnormalities observed following the use of AIs with the microarchitecture during bone remodeling appears to be imperative since this will provide additional information concerning the actual impact of structural abnormalities on bone strength during therapy with either anastrozole or letrozole. These data will help to re-evaluate the requirement for further screening of females receiving these medications.

Conclusion. Treatment with anastrozole and letrozole significantly affects bone metabolism, which appears to be directly associated with the biomechanical properties of bones. Future studies should investigate the interplay between bone pathological analysis and biomechanics among experimental animal models in order to further reveal the risks of bone fracture that arise from AI therapy.

\section{Acknowledgements}

The present study was supported by the IKY fellowships of excellence for postgraduate studies in Greece (Siemens program grant no. SPhD/11165/136). The authors wish to thank Dr Ioannis S. Vlachos (bioinformatician) and Mrs. Esmeralda Ntousi, Mr. Panagiotis Tsakiropoulos and Mr. Nicolaos Tsakiropoulos (technical staff) at the Laboratory of Experimental Surgery and Surgical Research 'N.S. Christeas' at the National and Kapodistrian University of Athens Medical School (Athens, Greece), for their assistance during the animal surgery and for their assistance with the biochemical analysis. A professional language-editing company, American Manuscript Editors (Seattle, WA, USA), proofread this manuscript.

\section{References}

1. Zhou F, Han S, Zhou N, Zheng W and Li P: Effects of modified Shu-Gan-Liang-Xue decoction combined with anastrozole on osteoblastic proliferation and differentiation of MC3T3-E1 cells. Mol Med Rep 11: 1639-1646, 2015.

2. Wilson S and Chia SK: Treatment algorithms for hormone receptor-positive advanced breast cancer: Applying the results from recent clinical trials into daily practice-insights, limitations, and moving forward. Am Soc Clin Oncol Educ Book 2013, doi: 10.1200/EdBook_AM.2013.33.e20.

3. Paridaens R, Thomas J, Wildiers J, Vermeiren P, Lobelle JP, di Salle E, Ornati G, Zurlo MG, Polli A, Lanzalone S and de Belder K: Safety, activity and estrogen inhibition by exemestane in postmenopausal women with advanced breast cancer: A phase I study. Anticancer Drugs 9: 675-683, 1998.

4. Van Asten K, Neven P, Lintermans A, Wildiers H and Paridaens R: Aromatase inhibitors in the breast cancer clinic: Focus on exemestane. Endocr Relat Cancer 21: R31-R49, 2014.

5. Khosla S, Melton LJ III and Riggs BL: The unitary model for estrogen deficiency and the pathogenesis of osteoporosis: Is a revision needed? J Bone Miner Res 26: 441-451, 2011.

6. Mazziotti G, Canalis E and Giustina A: Drug-induced osteoporosis: Mechanisms and clinical implications. Am J Med 123: 877-884, 2010.

7. McCloskey EV, Hannon RA, Lakner G, Fraser WD, Clack G Miyamoto A, Finkelman RD and Eastell R: Effects of third generation aromatase inhibitors on bone health and other safety parameters: Results of an open, randomised, multi-centre study of letrozole, exemestane and anastrozole in healthy postmenopausal women. Eur J Cancer 43: 2523-2531, 2007.

8. Hong AR, Kim JH, Lee KH, Kim TY, Im SA, Kim TY, Moon HG, Han WS, Noh DY, Kim SW and Shin CS: Long-term effect of aromatase inhibitors on bone microarchitecture and macroarchitecture in non-osteoporotic postmenopausal women with breast cancer. Osteoporos Int 28: 1413-1422, 2017.

9. Fusi C, Materazzi S, Benemei S, Coppi E, Trevisan G, Marone IM, Minocci D, De Logu F, Tuccinardi T, Di Tommaso MR, et al: Steroidal and non-steroidal third-generation aromatase inhibitors induce pain-like symptoms via TRPA1. Nat Commun 5: 5736, 2014.

10. Winters-Stone KM, Dobek J, Nail L, Bennett JA, Leo MC, Naik A and Schwartz A: Strength training stops bone loss and builds muscle in postmenopausal breast cancer survivors: A randomized, controlled trial. Breast Cancer Res Treat 127: 447-456, 2011.

11. Goss PE, Qi S, Cheung AM, Hu H, Mendes M and Pritzker KP: Effects of the steroidal aromatase inhibitor exemestane and the nonsteroidal aromatase inhibitor letrozole on bone and lipid metabolism in ovariectomized rats. Clin Cancer Res 10: 5717-5723, 2004.

12. Kumru S, Yildiz AA, Yilmaz B, Sandal S and Gurates B: Effects of aromatase inhibitors letrozole and anastrozole on bone metabolism and steroid hormone levels in intact female rats. Gynecol Endocrinol 23: 556-561, 2007.

13. Yonden Z, Aydin M, Alcin E, Kelestemur MH, Kutlu S and Yilmaz B: Effects of letrozole on bone biomarkers and femur fracture in female rats. J Physiol Biochem 65: 267-275, 2009.

14. Plourde PV, Dyroff M, Dowsett M, Demers L, Yates R and Webster A: ARIMIDEX: A new oral, once-a-day aromatase inhibitor. J Steroid Biochem Mol Biol 53: 175-179, 1995.

15. Crenshaw TD, Peo ER, Lewis AJ and Moser BD: Bone Strength as a trait for assessing mineralization in swine: A Critical Review of Techniques Involved. J Animal Science 53: 827-835, 1981. 
16. Czajka-Oraniec I and Simpson ER: Aromatase research and its clinical significance. Endokrynol Pol 61: 126-134, 2010.

17. Gonzales RJ, Ansar S, Duckles SP and Krause DN: Androgenic/estrogenic balance in the male rat cerebral circulation: Metabolic enzymes and sex steroid receptors. J Cereb Blood Flow Metab 27: 1841-1852, 2007.

18. Harada N, Sasano H, Murakami H, Ohkuma T, Nagura H and Takagi Y: Localized expression of aromatase in human vascular tissues. Circ Res 84: 1285-1291, 1999.

19. Kim JH, Cho HT and Kim YJ: The role of estrogen in adipose tissue metabolism: Insights into glucose homeostasis regulation. Endocr J 61: 1055-1067, 2014.

20. Tyrovola JB: The 'Mechanostat Theory' of Frost and the OPG/RANKL/RANK System. J Cell Biochem 116: 2724-2729, 2015.

21. Briot K and Roux C: Drug-induced osteoporosis: Beyond glucocorticoids. Curr Rheumatol Rep 10: 102-109, 2008.

22. Anastasilakis AD, Toulis KA, Polyzos SA and Terpos E: RANKL inhibition for the management of patients with benign metabolic bone disorders. Expert Opin Investig Drugs 18: 1085-1102, 2009

23. Weitzmann MN: The Role of Inflammatory Cytokines, the RANKL/OPG Axis and the Immunoskeletal Interface in Physiological Bone Turnover and Osteoporosis. Scientifica (Cairo) 2013: 125705, 2013

24. Folestad A, Ålund M, Asteberg S, Fowelin J, Aurell Y, Göthlin J and Cassuto J: Role of Wnt/ $\beta$-catenin and RANKL/OPG in bone healing of diabetic Charcot arthropathy patients. Acta Orthop 86 415-425, 2015.

25. Mezil YA, Allison D, Kish K, Ditor D, Ward WE, Tsiani E and Klentrou P: Response of bone turnover markers and cytokines to high-intensity low-impact exercise. Med Sci Sports Exerc 47: 1495-1502, 2015

26. Boyle WJ, Simonet WS and Lacey DL: Osteoclast differentiation and activation. Nature 423: 337-342, 2003.

27. Taylan A, Sari I, Akinci B, Bilge S, Kozaci D, Akar S, Colak A, Yalcin H, Gunay N and Akkoc N: Biomarkers and cytokines of bone turnover: Extensive evaluation in a cohort of patients with ankylosing spondylitis. BMC Musculoskelet Disord 13: 191, 2012.

28. Boyce BF and Xing L: Biology of RANK, RANKL, and osteoprotegerin. Arthritis Res Ther 9 (Suppl 1): S1, 2007.
29. Thomas GP, Baker SU, Eisman JA and Gardiner EM: Changing RANKL/OPG mRNA expression in differentiating murine primary osteoblasts. J Endocrinol 170: 451-460, 2001.

30. Ostrowska Z, Ziora K, Oświęcimska J, Swiętochowska E, Szapska B, Wołkowska-Pokrywa K, Dyduch A: RANKL/RANK/OPG system and bone status in females with anorexia nervosa. Bone 50: 156-160, 2012.

31. Wasilewska A, Rybi-Szuminska AA and Zoch-Zwierz W: Serum osteoprotegrin (OPG) and receptor activator of nuclear factor kappaB (RANKL) in healthy children and adolescents. J Pediatr Endocrinol Metab 22: 1099-1104, 2009.

32. Fyhrie DP and Vashishth D: Bone stiffness predicts strength similarly for human vertebral cancellous bone in compression and for cortical bone in tension. Bone 26: 169-173, 2000.

33. Goulet RW, Goldstein SA, Ciarelli MJ, Kuhn JL, Brown MB and Feldkamp LA: The relationship between the structural and orthogonal compressive properties of trabecular bone. J Biomech 27: 375-389, 1994.

34. Hooshmand S, Kern M, Metti D, Shamloufard P, Chai SC, Johnson SA, Payton ME and Arjmandi BH: The effect of two doses of dried plum on bone density and bone biomarkers in osteopenic postmenopausal women: A randomized, controlled trial. Osteoporos Int 27: 2271-2279, 2016.

35. Zioupos P and Currey JD: Changes in the stiffness, strength, and toughness of human cortical bone with age. Bone 22: 57-66, 1998.

36. Vegger JB, Brüel A, Sørensen TG and Thomsen JS: Systemic treatment with strontium ranelate does not influence the healing of femoral mid-shaft defects in rats. Calcif Tissue Int 98: 206-214, 2016.

37. Naruse K, Uchida K, Suto M, Miyagawa K, Kawata A, Urabe K, Takaso M, Itoman M and Mikuni-Takagaki Y: Alendronate does not prevent long bone fragility in an inactive rat model. J Bone Miner Metab 34: 615-626, 2016.

38. Gasser JA, Green JR, Shen V, Ingold P, Rebmann A, Bhatnagar AS and Evans DB: A single intravenous administration of zoledronic acid prevents the bone loss and mechanical compromise induced by aromatase inhibition in rats. Bone 39: 787-795, 2006.

39. Sengupta P: The laboratory rat: relating its age with human's. Int J Prev Med 4: 624-630, 2013 